\title{
Advancing in vitro diagnostics: an interview with Fernando Chaves
}

\author{
Fernando Chaves*,1 \\ ${ }^{1}$ Global Head, Clinical \& Scientific Affairs Ortho Clinical Diagnostics, Brooklyn, NY 11222, USA \\ *Author for correspondence: fernando.chaves@orthoclinicaldiagnostics.com
}

\begin{abstract}
Biography: Dr Fernando Chaves is a board-certified hematopathologist with over 15 years of experience in the diagnostic industry, where he has focused on studying, evaluating and bringing to market new laboratory tests for multiple medical conditions. He has worked with innovative researchers in multiple countries and across medical specialties, not only with new assays but also focusing on how to deploy multiparametric algorithms to improve the diagnostic performance of currently available tests. Dr Chaves serves as Global Head of Clinical and Scientific Affairs at Ortho Clinical Diagnostics.
\end{abstract}

First draft submitted: 26 July 2019; Published online: 10 September 2019

Keywords: cardiac troponin I • diagnostics $\bullet$ myocardial infarction $\bullet$ troponin assay $\bullet$ VITROS hs Troponin I

\section{This interview was conducted by Adam Price-Evans, Managing Commissioning Editor of Future Cardiology}

Could you please tell me about Ortho Clinical Diagnostics \& what your role within the company entails?

Ortho Clinical Diagnostics is focused on delivering in vitro diagnostics and serves two segments: clinical laboratories, with analyzers and assays that test for a variety of diseases, conditions and substances; and immunohematology, commonly referred to as blood banking, with technology and tests to ensure blood transfusion recipients receive appropriate and compatible blood.

I oversee Ortho's product portfolio from a medical and scientific perspective, ensuring that our assays are best positioned to meet unmet clinical needs, improve patient outcomes and lower overall healthcare costs, through better and faster diagnostics. We are also proud of our position in enabling patient safety, since our analyzers offer unique technology to prevent diagnostic errors caused by common interferences in the laboratory.

\section{Ortho Clinical Diagnostics has recently announced that the VITROS ${ }^{\circledR}$ High Sensitivity (hs) Troponin I} Assay is now CE marked \& available in the EU. Could you tell me a little about this? What are the intended clinical applications \& how will this benefit patients?

The VITROS hs Troponin I assay - the latest addition to Ortho's cardiology portfolio - is a tool designed for clinicians seeking improved strategies to more rapidly and accurately identify patients suffering from a myocardial infarction (MI). The assay also aids in quickly ruling out MI and identifying low-risk patients that may be safely discharged.

Considering the chaos of the Emergency Department, clinicians require the confidence that they are making the right decisions and are sending the patient on the right care pathway. Laboratories therefore need to be able to provide highly reliable results as quickly as possible in this urgent, time-critical clinical environment.

This assay has exceptional predictive value and high diagnostic accuracy that delivers high confidence rulein/rule-out clinical decisions. In addition, we have automated detection for hemolysis - when red blood cells are destroyed before testing - a cause of interference that occurs in 1 out of 20 blood samples coming from the Emergency Department. Disposable tips in the aspiration needles also helps avoid troponin from one patient being carried over to subsequent samples; and alkaline phosphatase is not required in our assay design.

The VITROS hs Troponin I assay is indicated to aid diagnosis of MI, which is the critical decision point. It is also indicated for risk stratification in acute cardiac syndrome patients up to 1 year, giving the clinician and the patient confidence that the decision is correct. 
Could you please describe this assay technology \& how it works specifically?

The VITROS hs Troponin I assay is built on Ortho's VITROS MicroWell Technology. Through proprietary enhanced chemiluminescence detection, this technology provides exceptional low-end sensitivity and precision, which are both critical for cardiac applications.

The assay implements an immunometric technique in this high-performance MicroWell format. Cardiac troponin I in the sample reacts with a prebound biotinylated antibody-specific coating and a labeled antibody conjugate. The assay uses monoclonal antibodies directed against the cardiac troponin I isoform specifically because it is more specific to myocardial injury than cardiac troponin T. This is important for patients with chronic kidney disease or musculoskeletal disease. The monoclonal antibodies bind to the more stable mid-fragment of the molecule, which is also a target for troponin auto-antibodies. This is important because these auto-antibodies can form large complexes with troponin (macrotroponin) and could lead to falsely elevated results. In studies which have shown up to $5 \%$ false-positive results in other instruments, caused by macrotroponin, our assay was not impacted at all. Additionally, our new assay runs on a broad range of Ortho analyzers already on the market.

\section{How does this assay advance current methods of detection?}

The VITROS hs Troponin I assay complies with all provisions of the International Federation of Clinical Chemistry and Laboratory Medicine definition for classification as a 'high sensitivity' troponin assay, enabling use with accelerated rule-in, rule-out diagnostic protocols. These algorithms are evolving specifically for high sensitivity assay use, and are allowing hospitals to more quickly and accurately identify and diagnose patients suffering from heart attacks, while more quickly ruling out those that are not.

With our updated design, we have made improvements to turnaround time, precision and - most importantly - to sensitivity. The assay represents a 10-fold improvement in limit of detection, which enables earlier detection of troponin levels elevated above the gender-specific 99th percentile decision point. Excellent precision at these clinical decision points enables detection of small changes in troponin levels, a key decision criterion in any acute MI diagnostic algorithm.

\section{Could you tell me a little about the results of clinical studies evaluating the assay?}

Data from clinical trials demonstrates the VITROS hs Troponin I assay's performance in early rule-in, rule-out algorithms based on overall 99th percentile. According to clinical study, the assay shows excellent prognostic value as an aid in the assessment of cardiac-related mortality/MI and all-cause mortality over 30 days, 6 months and 12 months.

Our ex-US clinical study looked at clinical performance of our assay using serial sample sets obtained from the Advantageous Predictors of Acute Coronary Syndromes Evaluation study collection. These are adjudicated samples from 12 sites in five European countries. They were collected at 0-, 1-, 2- and 3-h time point, with follow-up at 1, 6 and 12 months post-discharge.

From this study, we derived the area under the curve (AUC) of the receiver operating characteristic curve for delta values and for delta-plus-baseline at each time point. Our assay shows excellent separation between the MI and no-MI cohorts, with AUC above 0.943 at all time points. This AUC was statistically superior to the performance observed with other high sensitivity assays used for testing Advantageous Predictors of Acute Coronary Syndromes Evaluation samples in previous publications.

The study provided a wealth of data showing the excellent clinical performance of the assay. It also established the performance necessary to support the diagnostic and risk stratification claims. Altogether, the study provides the data needed to show compliance with all diagnostic algorithms, including 1, 2 and $3 \mathrm{~h}$ accelerated rule-in and rule-out algorithms from the European Society of Cardiology and American Heart Association.

How do you think the use of this assay will impact cardiac care?

Acute MI is an urgent, time-critical medical condition. We know that earlier detection saves lives. Compatibility of our assay with the hospital's accelerated rule-in/rule-out algorithm (whichever one they've chosen) means patients will benefit from better outcomes through earlier intervention. Clinicians benefit because they can have higher confidence in their decision making. Laboratories also benefit from the confidence that they have provided a highly reliable result, free from common interferences such as macrotroponin and hemolysis, ensuring fewer retests are required. Of course, hospitals benefit too, because earlier rule-out of patients not actually suffering a heart attack allows them to free up resources sooner, making room for the next patient who needs care. 


\section{Disclaimer}

The opinions expressed in this interview are those of the interviewee and do not necessarily reflect the views of Future Medicine Ltd (Future Science Ltd).

Financial \& competing interests disclosure

F Chaves is an employee of Ortho Clinical Diagnostics. The interviewee has no other relevant affiliations or financial involvement with any organization or entity with a financial interest in or financial conflict with the subject matter or materials discussed in the manuscript apart from those disclosed.

No writing assistance was utilized in the production of this manuscript. 
